\title{
Publisher Correction: Asymmetric O-propargylation of secondary aliphatic alcohols
}

Ren-Zhe Li, Da-Qi Liu and Dawen Niu(D)

Correction to: Nature Catalysis https://doi.org/10.1038/s41929-020-0462-9, published online 1 June 2020.

In the version of this Article originally published, the superscript numbers in equation (1) were incorrectly written as subscript numbers. The correct equation is: $\mathrm{d}[$ product $] / \mathrm{d} t=k[15]^{1}[16]^{0}[\mathrm{~B} 1]^{0}$. This has been corrected in the online versions of this Article.

Published online: 8 June 2020

https://doi.org/10.1038/s41929-020-0478-1

๑ The Author(s), under exclusive licence to Springer Nature Limited 2020 